\title{
Intensity Weighted Histogram Equalization Method for Night Vision
}

\author{
Ernesto Zamora Ramos \\ Computer Science Department, University of Nevada, Las Vegas, United States; \\ zamorara@unlv.nevada.edu
}

\begin{abstract}
This paper explores the possibility of utilizing histogram equalization on images captured in poor lighting conditions in order to expand their histogram dynamic range, enhancing their contrast and effectively provide night vision for such images. Then, some drawbacks of standard histogram equalization for dark images, caused mainly due to the clustering of pixels around the lowest intensities are exposed, and Enhanced Intensity Weighted Histogram Equalization is presented as a solution to obtain more realistic night vision images by incorporating the normalized weight of each pixel intensity into the calculations and spreading the histogram values to fill in the gaps, reducing noisy high frequency changes. This technology can be applied to new capture devices that detect the lack of illumination and engage Enhanced Intensity Weighted Histogram Equalization to provide low light capture, useful for surveillance, driving, medical imaging, and even space exploration.
\end{abstract}

Keywords: night vision, histogram equalization, contrast enhancement, image enhancement.

\section{Introduction}

It is well known that histogram equalization (HE) is a method utilized in digital image processing to enhance the contrast of an image. It works by expanding the dynamic range of the image histogram.

In the histogram for an image with poor contrast, it can be seen that all pixels are clustered close together around a few intensities. After applying histogram equalization to the image, it is observed how the pixels are no longer clumped together, but their intensities have been spread, trying to expand over the whole range of the histogram. This increased distance between intensities translates in an increased contrast for the image as it can be seen in figure 1.

Note that for this discussion, it is assumed, unless otherwise specified, that digital images have been converted to grayscale using the intensity value of each pixel, also known as luma or $Y$ component in the YUV color space, computed from the RGB color space as standardized by International Telecommunications Union in BT.601-7 [5]. 

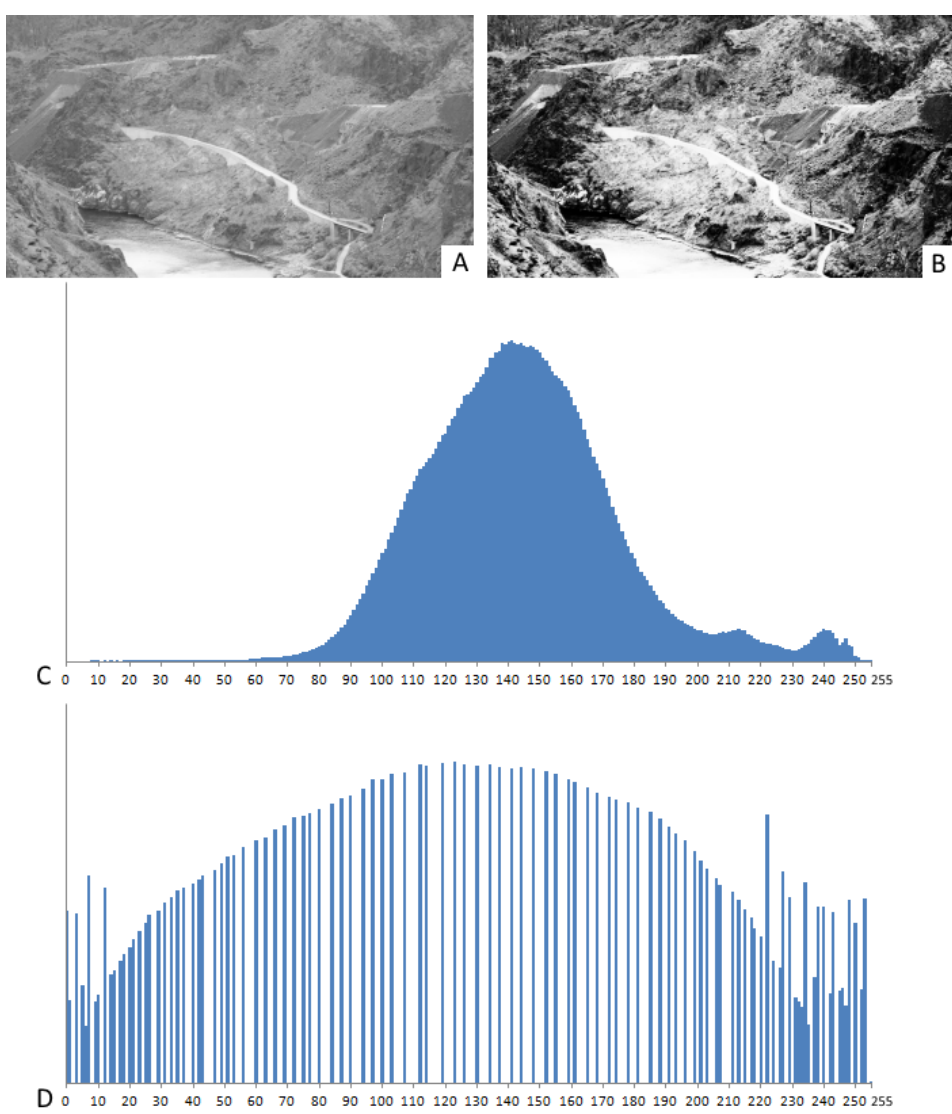

Figure 1. Poor contrast image before and after equalization. A) original image; B) image after histogram equalization; C) histogram of image A (notice how most pixels are clustered around a peak intensity);. D) histogram of image B (notice how the intensities have been spread apart).

HE accomplishes its goal by applying a non-linear transformation to the image in question. It computes the cumulative distribution function (CDF) of the histogram of the image, and then uses it as a look up table for the new pixel intensity values in the resulting image. Table I shows the definition of the classic HE algorithm.

Now, notice that the histogram for images captured with commercial digital cameras under poor illumination conditions is similar to that of an image with low contrast, but the intensities are closer to zero (see figure 2).

Table 1: Classic Histogram Equalization Algorithm [8]

Let I be the original image.

Let $\mathrm{I}(i)$ be the intensity of pixel $i$ in the image. $0 \leq \mathrm{I}(i) \leq \mathrm{X}_{h} . \mathrm{X}_{h}$ is the upper bound of the intensity.

Typically $X_{h}=255$.

Let $\mathrm{N}$ be the number of pixels in $\mathrm{I}$.

Let $\mathrm{H}$ be the histogram of $\mathrm{I}$. This is, $\mathrm{H}(x)$ is the number of pixels in I such that $\mathrm{I}(i)=x$.

Let $f(x)=\mathrm{H}(x) / \mathrm{N}$, be the probability function for a pixel in I to have intensity $x$.

Let $F(x)=\sum_{n=0}^{x} f(n)$, be the CDF for $f(x)$.

Then, the resulting image is defined as $\mathrm{I}^{\prime}(i)=\mathrm{X}_{h} \cdot F(\mathrm{I}(i))$.

If HE is applied to poorly illuminated images, the result is a clearer picture. The little energy captured is amplified, lighting up the original image, and making the shapes visible to the human eye (see figure 3). 
It is clear that if image capture devices are equipped with means of detecting low illumination and small dynamic ranges in the histogram of the capture, the subsequent captures can be subject to some variant of HE to improve contrast and effectively perform night vision via software. Such devices can be used as an alternative option to current and more expensive night vision devices such as infrared or

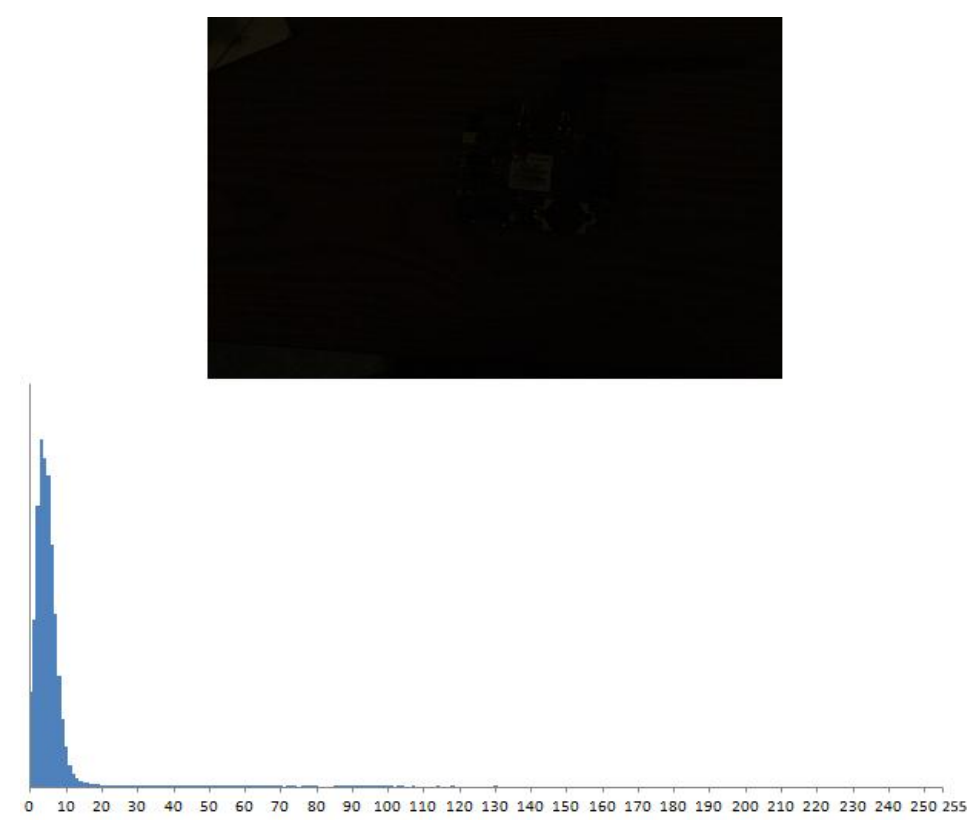

Figure 2. Image captured with poor illumination and its corresponding histogram. Notice how the intensities are clustered together, akin to an image with poor contrast.

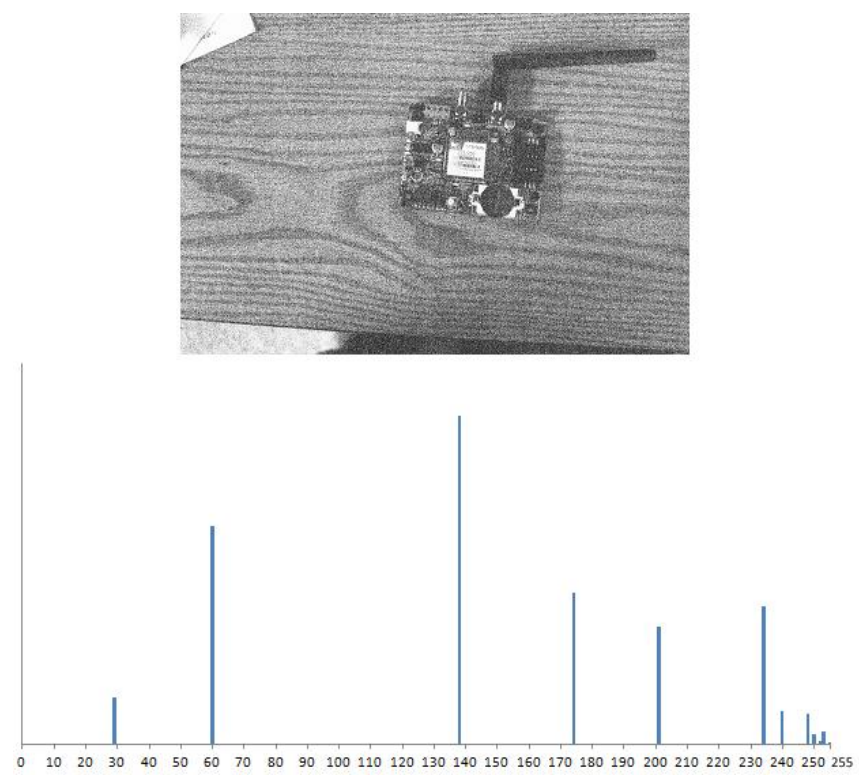

Figure 3. Image captured with poor illumination and its corresponding histogram. Notice how the intensities are clustered together, akin to an image with poor contrast.

thermal cameras and to improve current camera technology, ubiquitous in automobiles $[4,6]$, cell phones and other wearables. These cameras can also be very useful for surveillance, nocturnal observations such as wildlife and deep ocean exploration, medical imaging enhancement, search and rescue, and even space exploration. 
However, after several experiments, it can be noticed that HE fails to expand the histogram to the whole dynamic range in dark images. Observe that an undesired artifact of applying HE as an alternative to enhance poorly illuminated scenes is the washed out, overly bright nature of the result. The reasons for this behavior were determined and a variation of the algorithm was developed that results in sharper images with better contrast where the whole range of the histogram is utilized.

The rest of this paper discusses some of the work done relating to software night vision and $\mathrm{HE}$, followed by the exposition of the proposed modifications to HE and enhancements; concluding with some experimental examples of the improved results.

\section{Related Work}

Even though standard HE offers a direct alternative for the enhancement of dark images, in many occasions the resulting images are too bright, or washed out, so, there have been several works that propose improvements upon this technique in general, and for the purpose of night vision.

There are many variations to the HE algorithm. Many of these try to increase the contrast and dynamic range of an image while preserving the brightness or expected intensity value with the purpose of applying it to images with low contrast, but not specifically for poorly illuminated images.

In their work "Contrast Enhancement Using Brightness Preserving Bi-Histogram Equalization" [10], the authors explain a popular variation of standard $\mathrm{HE}$, called Bi-Histogram Equalization (Bi-HE). This is one of several variations to $\mathrm{HE}$ that pursues to maintain the image average intensity while expanding the dynamic range. Bi-HE seeks to divide the image histogram into two histograms separated by the mean value. standard HE is then performed on each sub-histogram. Methods like this are introduced in an attempt to make HE viable in consumer electronics by lessening the artifact of brightness change in an image after equalizing it.

Chen and Ramli [9] propose a variation of Bi-histogram equalization where they apply Bi-HE recursively. Each new section of histogram delimited by its bounds or means is subjected to Bi-HE once again, and so on.

Another variation divides the image into regular regions of equal area and apply $\mathrm{HE}$ to each region so that the variance of the intensity is smaller in the locality and does not affect the result as drastically as the global variance [8]. The result, however, is a patchy image with unnaturally bright areas.

While histogram equalization methods that preserve brightness could be useful to enhance images with poor contrast, they may not be as appropriate to utilize for poorly illuminated images because the intention is to increase their contrast as well as to brighten them up. These methods also tend to suffer from the same downside as standard HE when applied to dark images as explained later in this paper.

However, histogram equalization has only been used in a limited amount of work as a night vision alternative. Many authors have also realized that standard HE and variations used in other types of images need to be modified in order to provide better results for dark images.

In his thesis work [2], Teo realizes that HE, or one of its variants, can be applied to night vision images to enhance their contrast and improve further on the quality of visuals. The author, however, applies the technique to images already captured with night vision or thermal imaging devices, so, the effect of $\mathrm{HE}$ on these images is not as pronounced as when applied directly to the original dark images.

In their paper "New image enhancement algorithm for night vision," [11] the authors propose a combination of $\mathrm{HE}$ and contrast enhancement to improve upon standard HE when used for dark 
images after realizing the unnatural increase in brightness in resulting images when applied for night vision.

Sapkota [7] shows in his work the application of the concept, already explaining the possibility of building a capture device that utilizes HE to provide night vision for low light environments. He goes on to explain Incremental Histogram Equalization to look for the optimal upper bound of dynamic range expansion of the histogram where the resulting image would more closely resemble the well illuminated version. The author, however, still uses the standard HE algorithm from table I to obtain the night vision result, just varying the upper bound $X_{h}$ to find the peak of signal to noise ratio.

In most of these papers, the variations to the standard HE provide some improvement on the resulting images, however, almost none addressed the issue that $\mathrm{HE}$, in fact, does not expand the histogram to the whole dynamic range for poorly illuminated images as it does for better lit images.

\section{Proposed Intensity Weighed Histogram Equalization}

Observe how the result from applying HE to a dark image usually looks washed out, and overall, too bright (see figure 3 ), making some details close to the higher intensities practically indistinguishable. This is one of the undesired artifacts introduced by the equalization process. Studying the histograms of resulting images closely, the cause of this undesired effect can be identified and the technique can be adjusted accordingly.

On dark images, the zero and close to zero intensities are predominant, and their effect is what causes the phenomenon highlighted in the histogram in figure 4. Applying classic HE to most images will cause this effect. Notice from the definition of the algorithm in table 1 that if there are pixels with zero intensity, then $F(0)=u>0$. So, in the new image all pixels will be $I^{\prime}(i) \geq u \cdot X_{h}$. This means that the gap highlighted in figure 4 is of magnitude $u$ intensities. The main problem with this incident is that classic HE will not map successfully the pixels to the dynamic range of the histogram when applied to dark images. Also, no matter what, pixels that would actually have zero intensity, will be given an artificial value.

Intensity Weighted Histogram Equalization (IWHE) is the proposed solution to this problem. It is a variation of the standard $\mathrm{HE}$, and it is also a global equalization method (i.e. it operates over the whole image instead of smaller regions). The main modification in IWHE consists on replacing the computation of the resulting image with the formula:

$$
\mathrm{I}^{\prime}(i)=\min \left(\mathrm{X}_{h} \cdot F(\mathrm{I}(i)) \cdot \frac{\mathrm{I}(i)}{\text { limit } Y}, \mathrm{X}_{h}\right)
$$

Where $0<$ limit $Y \leq X_{h}$ is the intensity value where a desired percentage of the pixels have already appeared.

Experimental results suggest that the best quality images are obtained when the value for limitY satisfies: 


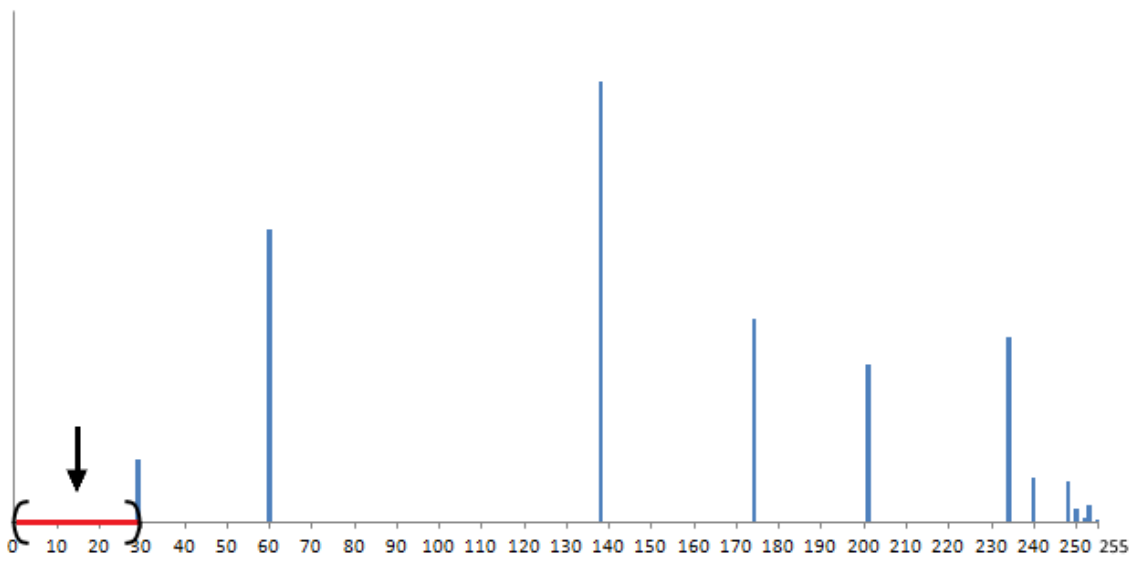

Figure 4. Lapse in intensities before the lowest intensity appears in the histogram for an equalized poorly illuminated image using classic $\mathrm{HE}$.

$$
0.97 \leq \frac{\sum_{n=0}^{\text {limit } Y} H(n)}{N} \leq 0.99
$$

That is, limitY is the intensity value in the histogram where $97 \%$ to $99 \%$ of all pixels in the original image have been accounted for. The smallest intensity where $100 \%$ of pixels have been counted is ideal to spread all pixel intensities across the whole dynamic range $\left(0, X_{h}\right)$, but the image may still be a little dark because there is usually a small amount of pixels spread among the intensities after $99 \%$ of pixels have been counted. The outlying $1 \%$ of the pixels should be set to maximum intensity while spreading the rest.

The parameter limitY, within reasonable values, can be used, in effect, to control the brightness of the resulting image. It inversely affects the overall brightness. Larger values offer more spread of the intensities, but reducing the brightness. Smaller values can cause loss of information because too

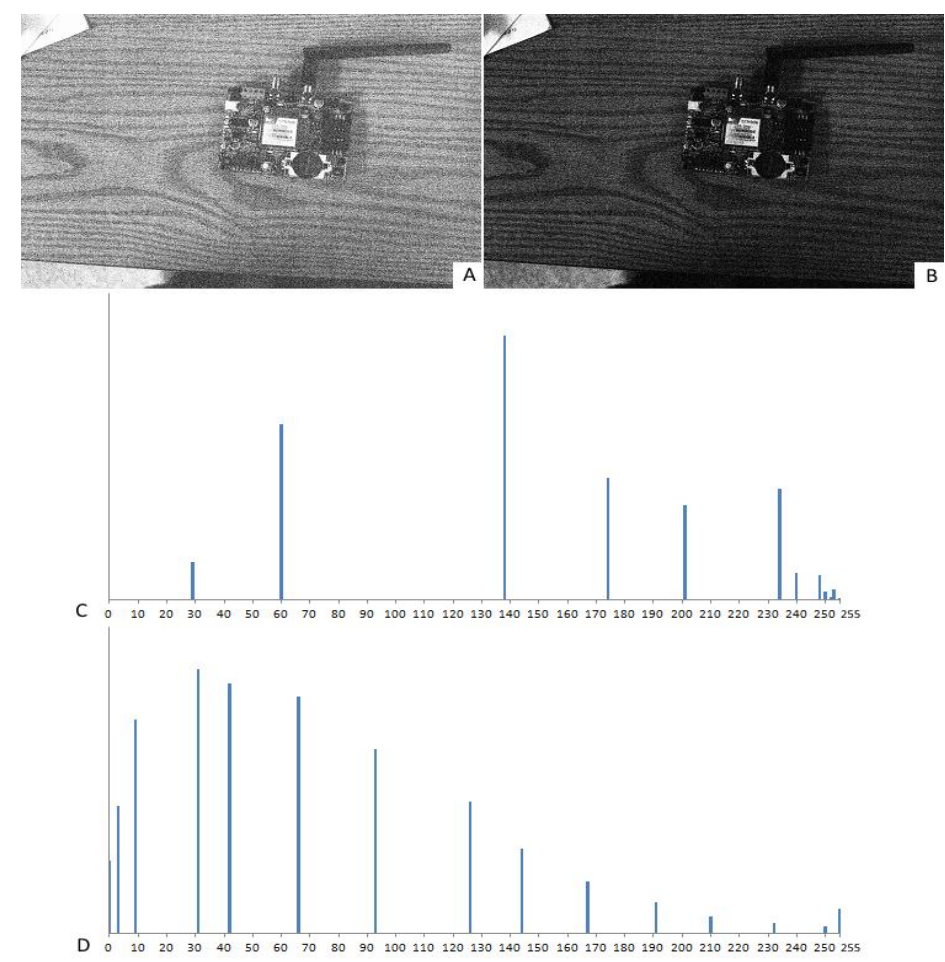

Figure 5. A) classic histogram equalization of image in figure 2; B) Intensity Weighted Histogram Equalization of same image; C) histogram for image A; D) histogram for image $B$. 
many pixels will be moved to full intensity.

The expression $\frac{\mathrm{I}(i)}{\text { limitY }}$ used in the new formula to compute the resulting intensity considers the normalized weight of the pixel intensity when expanding the dynamic range of the histogram. The result, as seen in figure 5 , is an image that looks more natural, no longer washed out, more detailed, and arguably less noisy than its classic histogram equalized counterpart. Even the text and bar codes engraved on the chip are visible and readable. Observe that the resulting histogram has the values spread over the whole range instead of starting with a constant gap, and the pixels are distributed over more intensities. The new equalization also ensures that pixels with originally zero intensity, remain at zero. Black pixels had no energy captured by the camera, and thus it is artificial to give them values too high. This result was achieved after applying IWHE with a limitY that satisfies $\frac{\sum_{n=0}^{\text {limit } Y} H(n)}{N}=0.98$ (refer to equation (2)).

\section{Enhanced Intensity Weighed Histogram Equalization}

As it can be seen in figure 5, when a poorly illuminated image is enhanced with IWHE, the resulting histogram has expanded to take over the whole dynamic range and it retains a shape similar to the original image histogram, thus giving the resulting image a more natural look.

Note that the resulting histogram is still a comb containing gaps between intensity values giving the image sharp jumps in intensity among pixel regions. To improve upon this, the pixels can be distributed around their representative intensity value in a normal-distribution-like pattern.

Buckets are created around each individual intensity in the resulting histogram of IWHE. For each bucket, the left limit is defined to be the value halfway between the representative intensity and the neighbor intensity to the left. The right limit is defined in a similar manner. Afterwards, the limits of each bucket are extended based on its size to overlap with adjacent buckets (i.e., larger buckets receive a larger extension to each side). See figure 6.

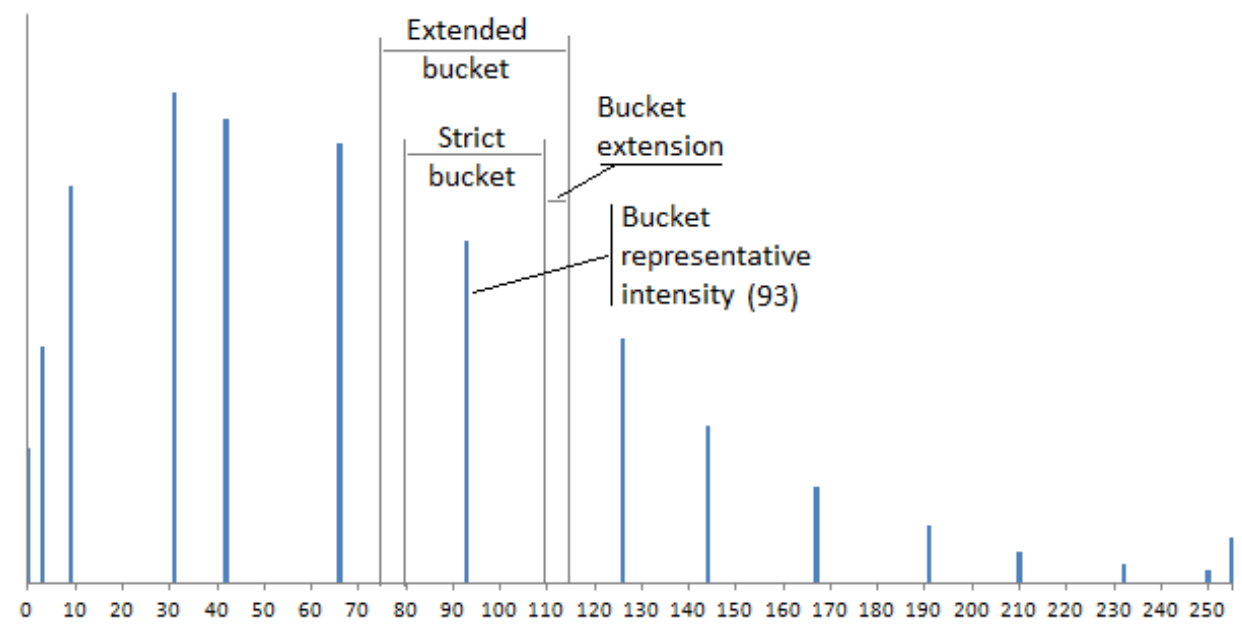

Figure 6. Depiction of a bucket around a representative intensity (in this case, intensity value 93). Strict bucket limits are the halfway values between intensities. The extended bucket is the final bucket and is computed by adding the bucket extension to each side of the strict bucket to achieve overlap between buckets. 

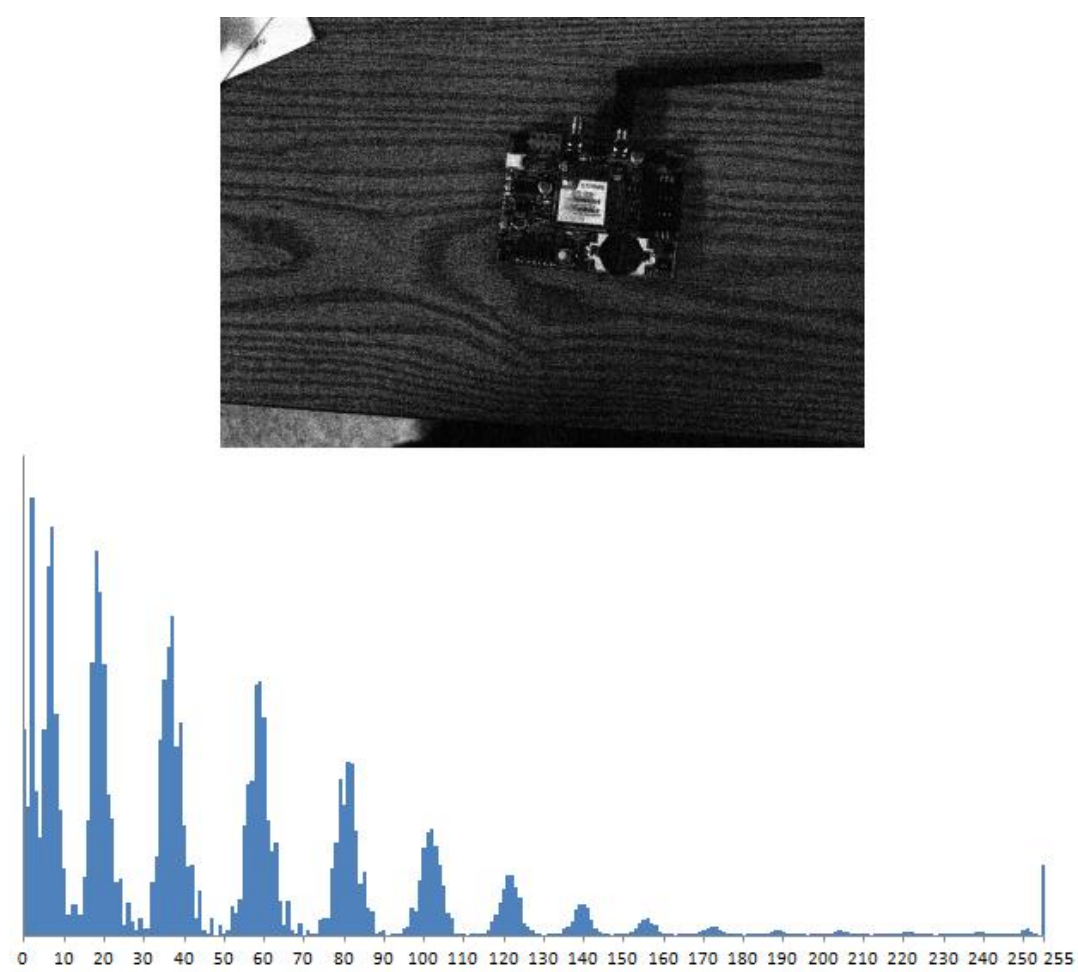

Figure 7. Result of applying Enhanced IWHE to the poorly illuminated image from figure 2. Notice how the histogram distribution has values over the whole range without holes.

The $\mathrm{N}_{8}$ vicinity of a pixel $p$ is the collection of pixels (including $p$ ) in the $3 \times 3$ matrix of pixels centered at $p$. The IWHE result is passed through a filtering mechanism where a new intensity value $I_{1}(p)$ is computed based on the intensity of the pixels in the $\mathrm{N}_{8}$ vicinity of $p$ (for the experiments, the average of the intensities was used, but any other filter that can spread the values in the bucket as defined works as well).

$$
\mathrm{I}_{1}(p)=f\left(\mathrm{I}\left(\mathrm{N}_{8}(p)\right)\right)
$$

Then, the range $\mathfrak{R}$ delimited by $\min \left(I\left(p_{i}\right)\right)$ and $\max \left(I\left(p_{i}\right)\right)$ is mapped linearly to the range $\mathfrak{R}$ for the bucket corresponding to the intensity of $p$. The intensity value for $p$ in the final image, $I^{\prime}(p)$, is the value $I_{1}(p)$ mapped from $\mathfrak{R}$ to $\mathfrak{R}$ :

$$
\mathrm{I}^{\prime}\left(\mathrm{I}_{1}(p)\right): \mathfrak{R} \rightarrow \mathfrak{R}^{\prime}
$$

Figure 7 shows the final result and histogram obtained after applying this method to the dark image in figure 2. The images enhanced with this method present reduced noise on areas where uniform intensity is expected due to the attenuation of high frequency regions.

\section{Experimental Results}

A collection of pictures was captured with two different commercially available cameras with no night vision capabilities (other than flash) under poor light conditions. The variety of images included night time landscapes as well as objects captured in a closed room without illumination. 

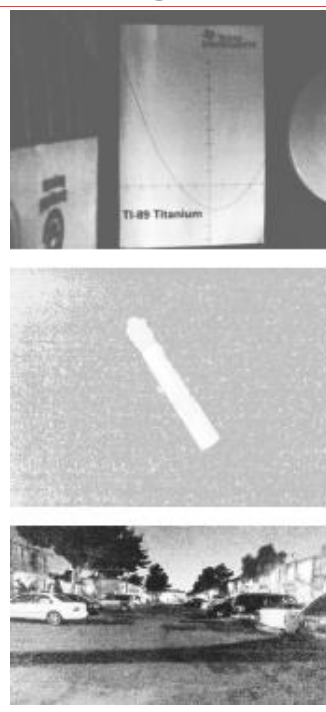
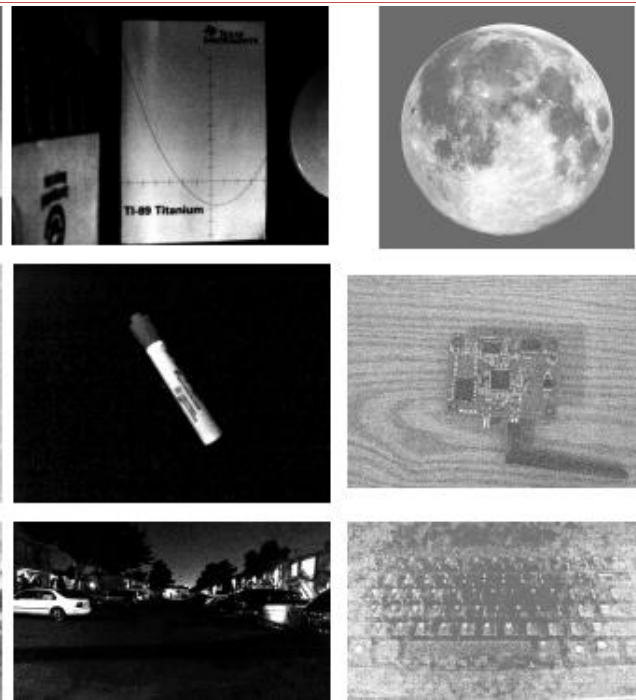
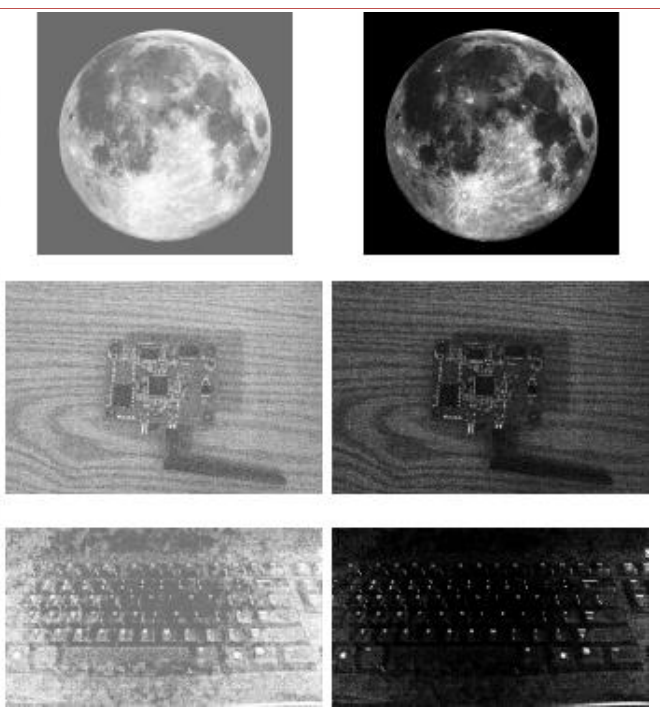

Figure 8. Visual comparison between standard HE and Enhanced IWHE. Images are arranged in pairs where the left image of the pair was obtained through the application of standard HE to a poorly illuminated scene and the right image was obtained through the application of Enhanced IWHE to the same image. Topleft: book; top-right: the moon (original photo obtained from NASA website); center-left: marker; center-right:

circuit board; bottom-left: apartment complex parking lot at midnight; bottom right: computer keyboard.

Figure 8 shows a visual comparison between the application of HE and Enhanced IWHE to improve some of the poorly illuminated images captured. The original images are omitted because their poor illumination offers no meaningful detail. With both cameras, the obtained pictures were nothing more than dark images much like figure 2. Notice how images treated with Enhanced IWHE retain a more realistic look and details than their washed out, surreal HE counterparts.

Available upon request are the C\# program used for the experiments, a Windows Store App demonstrating proof of concept, and the original version of all images utilized for this study.

\section{Conclusions}

Histogram equalization is an inexpensive method to increase the contrast in an image that can be utilized to enhance visibility in poorly illuminated scenes, effectively providing night vision capabilities. Although, dark images receive improved lighting when equalized, the results look washed out and unnatural, due to the concentration of pixels with zero intensities, and their histogram shows a comblike shape with large gaps between intensities. The introduction and application of Enhanced Intensity Weighted Histogram Equalization as a global equalization technique tackles these problems by considering the normalized weight of each pixel when equalizing the image, expanding the histogram to utilize the whole dynamic range, producing crispier, more natural looking, more detailed and less noisy results than standard histogram equalization for dark images.

This technology can be embedded in capture devices that engage Enhanced IWHE when a small dynamic range is detected on the input image histogram, with values clustered close to lower intensities, as a less expensive alternative to other night vision technologies, such as infrared cameras. This will provide enhanced images from captures in low light environments, useful in cameras employed for surveillance, driving, search and rescue, observation of wild life at night or deep ocean, medical imaging enhancement, and many others. 


\section{REFERENCES}

[1] A. Waxman, D. Fay, P. Ilardi, D. Savoye, R. Biehl, D. Grau, "Sensor Fused Night Vision: Assessing Image Quality in the Lab and in the Field," 9th International Conference on Information Fusion, 2006, pp. 1-8.

[2] C. K. Teo, "Digital Enhancement of Night Vision and Thermal Images," Thesis, Naval Postgraduate School. Monterey, California, USA. 2003.

[3] D. Fay, P. Ilardi, N. Sheldon, D. Grau, R. Biehl, A. Waxman, "Real-time image fusion and target learning \& detection on a laptop attached processor," International Conference on Information Fusion, 2005, pp. 499-506.

[4] F. Schule, R. Schweiger, K. Dietmayer, "Augmenting Night Vision Video Images with Longer Distance Road Course Information," Intelligent Vehicles Symposium (IV), 2013 IEEE, pp. 1233-1238.

[5] International Telecommunications Union. (2015). BT.601: Studio encoding parameters of digital television for standard 4:3 and wide screen 16:9 aspect ratios [Online]. Available: http://www.itu.int/rec/R-REC-BT.601-7-201103-1/en

[6] M. Serfling, O. Loehlein, R. Schweiger, K. Dietmayer, "Camera and Imaging Radar Feature Level Sensorfusion for Night Vision Pedestrian Recognition," Intelligent Vehicles Symposium, 2009 IEEE, pp. 597-603.

[7] N. Sapkota, "Real Time Digital Night Vision Using Nonlinear Contrast Enhancement," MS Thesis. UNLV Theses/Dissertations/Professional Papers/Capstones. 2013.

[8] R. C. Gonzalez and R. E. Woods, "Histogram Equalization," in Digital Image Processing, 3rd ed., UP, India: Prentice Hall, 2007, pp. 122-128.

[9] S. D. Chen and R. Ramli, "Contrast Enhancement using Recursive Mean-Separate Histogram Equalization for Scalable Brightness Preservation," IEEE Trans. Consumer Electronics. vol. 49, no. 4, pp. 1301-1309, 2003.

[10] Y.T. Kim, "Contrast Enhancement Using Brightness Preserving Bi-Histogram Equalization," IEEE Trans. Consumer Electronics, vol. 43, no. 1, pp. 1-8, Feb. 1997.

[11] Z. Yu, W. Xiqin, and P. Yingning, "New image enhancement algorithm for night vision," ICIP 99. Proceedings. 1999 International Conference on Image Processing, vol. 1, pp. 201-203, 1999. 